\title{
Prediction of Liver Fibrosis Using CT Under Respiratory Control: New Attempt Using Deformation Vectors Obtained by Non-rigid Registration Technique
}

\author{
AKIHIRO NISHIE ${ }^{1}$, SADATO AKAHORI $^{2}$, YOSHIKI ASAYAMA ${ }^{1}$, KOUSEI ISHIGAMI $^{1}$, \\ YASUHIRO USHIJIMA ${ }^{1}$, DAISUKE KAKIHARA ${ }^{1}$, TOMOHIRO NAKAYAMA $^{1}$, YUKIHISA TAKAYAMA ${ }^{3}$, \\ NOBUHIRO FUJITA ${ }^{1}$, KOICHIRO MORITA ${ }^{1}$, KEISUKE ISHIMATSU ${ }^{1}$, SEIICHIRO TAKAO $^{1}$, \\ TOMOHARU YOSHIZUMI ${ }^{4}$, KENICHI KOHASHI ${ }^{5}$, YUANZHONG LI ${ }^{2}$ and HIROSHI HONDA ${ }^{1}$ \\ ${ }^{1}$ Department of Clinical Radiology Graduate School of Medical Sciences, Kyushu University, Fukuoka, Japan; \\ ${ }^{2}$ Imaging Technology Center, Research and Development Management Headquarters, \\ FUJIFILM Corporation, Tokyo, Japan; \\ ${ }^{3}$ Department of Radiology Informatics and Network, \\ Graduate School of Medical Sciences, Kyushu University, Fukuoka, Japan; \\ ${ }^{4}$ Department of Surgery and Science, Graduate School of Medical Sciences, Kyushu University, Fukuoka, Japan; \\ ${ }^{5}$ Department of Anatomic Pathology, Graduate School of Medical Sciences, Kyushu University, Fukuoka, Japan
}

\begin{abstract}
Aim: To investigate whether liver fibrosis can be predicted by quantifying the deformity of the liver obtained based on computed tomographic (CT) images scanned under respiratory control. Materials and Methods: For dynamic CT of 47 patients, portal venous and equilibrium phases were scanned during inspiration and expiration, respectively. After rigid registration of the two images, non-rigid registration of the liver was performed, and the amount and direction of each voxel's shift during non-rigid registration was defined as the deformation vector. The correlation of each CT parameter for the obtained deformation vectors with the pathologicallyproven degree of liver fibrosis was assessed using Spearman's rank correlation test. Receiver operating characteristic curve analysis was conducted for prediction of liver fibrosis. Results: The standard deviation, coefficient of variance $(C V)$ and skewness were significantly negatively correlated with the degree of liver fibrosis ( $p=0.030,0.009$ and 0.037, respectively). Of these measures, $C V$ was best correlated and significantly decreased as liver fibrosis progressed (rho=-0.376). CV showed accuracies of 66.0-70.2\%, and the
\end{abstract}

Correspondence to: Akihiro Nishie, MD, Department of Clinical Radiology, Graduate School of Medical Sciences, Kyushu University, 3-1-1, Maidashi, Higashi-ku Fukuoka, 812-8582, Japan. Tel: +81 926425695, Fax: +81926425708, e-mail: anishie@ radiol.med.kyushu-u.ac.jp

Key Words: Multidetector-row computed tomography, liver, fibrosis, elastography, stiffness. areas under curves were 0.654-0.727 for prediction of fibrosis of grade F1 or greater, F2 or greater, F3 or greater and F4 fibrosis. Conclusion: The deformation vector is a potential CT parameter for evaluating liver fibrosis.

Liver fibrosis is a factor suggesting the degree of liver injury and is often considered when determining the indication for antiviral therapy (1-3). Without proper management, the progression of liver fibrosis consequently leads to cirrhosis, which increases morbidity and mortality caused by the development of hepatocellular carcinoma, as well as portal hypertension and hepatic insufficiency (4). To date, liver fibrosis has mostly been assessed by liver biopsy. However, biopsy carries an inherent risk of complications such as hemorrhage and infection. In addition, both the frequency of diagnostic disagreement among pathologists and the possibility of sampling error introduce serious uncertainty to liver biopsy results. For all these reasons, it would be of great clinical value if the histology of the liver parenchyma could be determined using an objective and non-invasive imaging modality.

Several methods to predict liver fibrosis according to changes in morphology, texture and perfusion have been reported on ultrasound (US), computed tomography (CT) and magnetic resonance imaging (MRI) (5-8). When limited to MRI, diffusion-weighted imaging and gadoxetic acidenhanced MRI also have the potential to predict liver fibrosis $(9,10)$. 'Stiffness' is a surrogate marker for liver fibrosis. On US and MRI, stiffness is predicted with elastography, which enables the non-invasive measurement 
of tissue mechanical properties through observation of shear-wave propagation in the tissue of interest (11). MR elastography is particularly considered to have the highest diagnostic performance for staging liver fibrosis (5). Although elastography is a useful tool for predicting liver fibrosis, no similar analytical method based on stiffness exists for CT. However, the liver parenchyma has been shown to be pushed in inferior and anterior directions by the lower lung lobes (12) and to change shape between expiration and inspiration (13). We hypothesized that the degree of these changes might serve as an indicator of stiffness, and ultimately as an indicator of liver fibrosis, if such a deformity could be quantified.

In this study, we investigated whether liver fibrosis can be predicted by quantifying the deformity of the liver obtained based on CT images scanned under respiratory control, that is, scanned during inspiration and expiration.

\section{Materials and Methods}

Patients. This study was approved by the Institutional Review Board of our hospital (no. 30-180). The requirements for informed consent were waived for this retrospective study. Referring to the medical data recorded at our hospital, 124 and 107 patients underwent dynamic CT for the liver with respiratory control, and hepatic resection for liver tumor or liver transplantation between May 2017 and March 2018, respectively. We enrolled 82 patients who underwent both dynamic CT for the liver with respiratory control and hepatic resection for liver tumor or liver transplantation. Of these, one radiologist with 24 years of experience, who was unaware of the pathological results, excluded patients with presence of perihepatic ascites $(n=11)$, poor respiratory control during CT scanning ( $n=7)$, or with history of lower lobectomy of the lung $(n=2)$ or right lobectomy of the liver $(n=1)$. We considered that ascites would disturb the external force by inspiration to the liver. Poor respiratory control was defined as less than $2 \mathrm{~cm}$ difference in height of the top of the diaphragm between inspiration and expiration. We also considered that in patients with history of major resection of the liver and the lung, such as lobectomy, the external force by inspiration would not be sufficiently transmitted to the liver. Furthermore, 14 patients were excluded due to inaccurate contour extraction of the liver in the process of image analysis described below. Inaccurate contour extraction of the liver suggested that the contour of the liver could not be accurately recognized during the image analysis and that the degree of the liver deformity would not be accurately evaluated. Consequently, the remaining 47 patients included 33 men and 14 women (age range $=39-85$ years; mean age $=67$ years). The underlying liver disease was hepatitis B viral infection in seven cases, hepatitis $\mathrm{C}$ viral infection in 15, alcoholic liver disease in four, non-alcoholic steatohepatitis in three, autoimmune hepatitis in one case, unknown etiology in five, and no apparent cause in 12 cases. The grading of liver dysfunction was pre-operatively evaluated based on the Child-Pugh classification. Thirty-seven, eight, and two patients were categorized into grade $\mathrm{A}, \mathrm{B}$, and $\mathrm{C}$, respectively. The liver tumors resected were hepatocellular carcinoma in 31 cases, intrahepatic cholangiocellular carcinoma in three, liver metastasis in four, focal nodular hyperplasia in one case and epithelioid hemangioendothelioma in one case; seven cases had no tumor. The range of tumor size was 10 to $85 \mathrm{~mm}$.
CT imaging. CT examination and surgery were performed within 1 month for all patients, and no patient underwent treatment during that period. Preoperative CT examinations were performed with a 320-slice multidetector-row CT scanner (Aquilion ONE; Toshiba Medical Systems, Nasu, Japan) with a gantry rotation time of $0.5 \mathrm{~s}$. Intravenous nonionic contrast material $(1.62 \mathrm{ml} / \mathrm{kg}$; $\max 100 \mathrm{ml})$ containing $370 \mathrm{mg}$ iodine/ml (Iopamidol, Iopamiron 370; Bayer Schering Pharma, Osaka, Japan) was injected over a 30 -s period by means of an automated power injector. The scan parameters were as follows: Section collimation, $0.5 \mathrm{~mm}$; helical pitch, 0.813 ; fieldof-view, $320 \mathrm{~mm}$; matrix, 512; auto $\mathrm{mA}$; and $120 \mathrm{kVp}$. The section thickness was $1.0 \mathrm{~mm}$. The bolus tracking technique was used to determine the timing of the dynamic study; two continuous hepatic arterial phases were obtained during a single breath-hold $15 \mathrm{~s}$ after a 100-HU elevation of density in the descending aorta. The portal venous and equilibrium phases were acquired at 60 and $240 \mathrm{~s}$, respectively, after the initiation of contrast material injection. Precontrast, hepatic arterial and portal venous phases were scanned during inspiration, while the equilibrium phase was acquired during

Figure 1. Details of image analysis. Image analysis had three stages as described below. Axial, sagittal and coronal images at each step are shown. A: Contour extraction of the liver using computed tomographic (CT) images scanned during inspiration and expiration. Based on CT values of air, the human body was recognized. Next, a CT slice showing the top of the liver was predicted by counting voxels suggestive of air in the body. Lastly, according to the CT values themselves and difference in $C T$ values between adjacent voxels, the liver was extracted. A data analyst evaluated the accuracy of contour extraction. B: Rigid registration of CT images scanned during inspiration and expiration. Firstly, the spinal contours of the CT images scanned during inspiration and expiration were matched. Conversion parameters for rigid registration (only parallel shift and rotation) were applied to CT images scanned during expiration. Coincidence of the extracted liver after rigid registration with the liver extracted from CT images scanned during inspiration was calculated. Conversion parameters for rigid registration were repeatedly updated so that coincidence was maximized. Finally, the CT images scanned during expiration after optimal rigid registration were obtained. However, the livers extracted from the CT images scanned during inspiration and expiration still showed a morphological difference. C: Non-rigid registration of CT images scanned during inspiration and expiration and generation of deformation vector field. The non-rigid registration was performed based on B-spline and diffeomorphic conversion models. Conversion parameters for non-rigid registration were applied to the CT images scanned during expiration after optimal rigid registration. Coincidence of the extracted liver after non-rigid registration with the liver extracted from CT images scanned during inspiration was calculated. Conversion parameters for non-rigid registration were repeatedly updated so that coincidence was maximized. Finally, the CT images scanned during expiration with optimal non-rigid registration were obtained. The livers extracted from the CT images scanned during inspiration and expiration showed a morphological coincidence with high precision at this stage. The amount and direction of shift for each voxel during the non-rigid registration was defined as a deformation vector; that is, a shift of each voxel between the CT images scanned during expiration after optimal rigid registration and the CT images scanned during expiration after optimal non-rigid registration. A deformation vector field, comprising deformation vectors for all voxels, was generated. Here, deformation vectors were simply described at intervals of $1.5 \mathrm{~cm}$. 
A

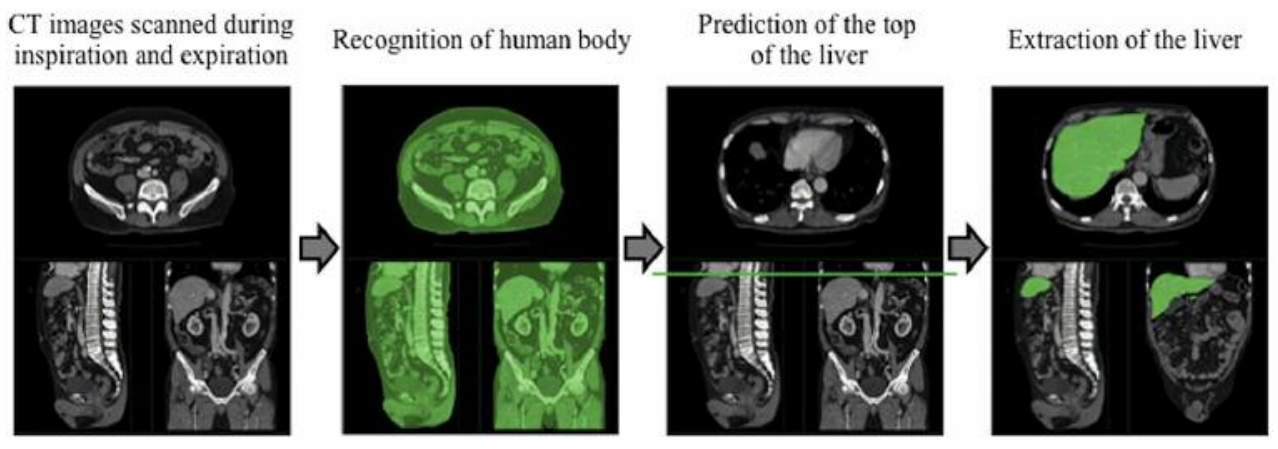

B

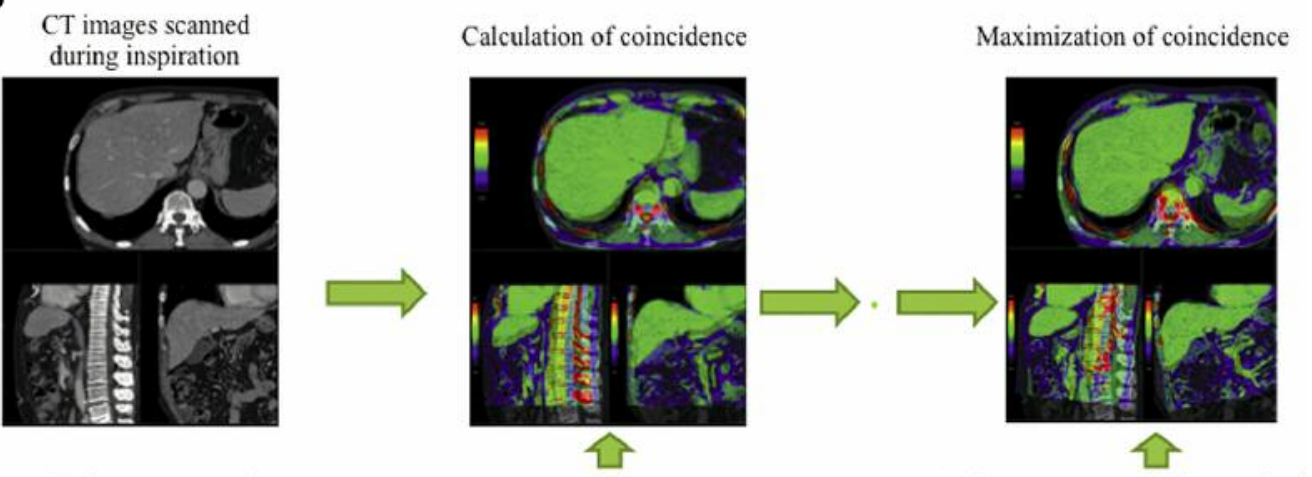

CT images scanned during expiration

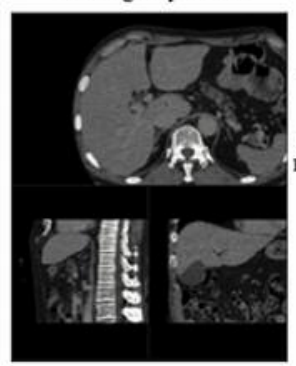

CT images scanned during expiration after rigid registration

$\mathrm{CT}$ images scanned during expirati

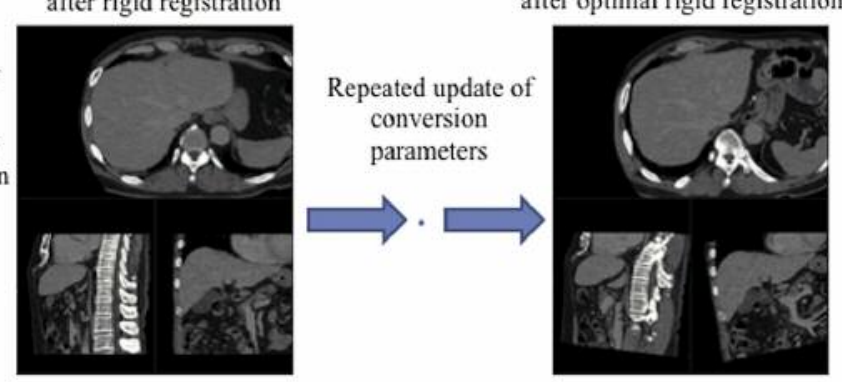

C

CT images scanned
during inspiration

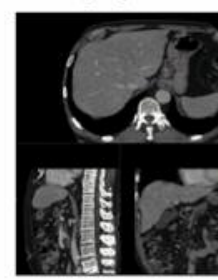

Application of conversion parameters for rigid registration

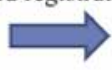
conversion parameters

images scanned during expiration after optimal rigid registration

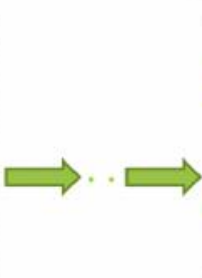

Maximization of coincidence

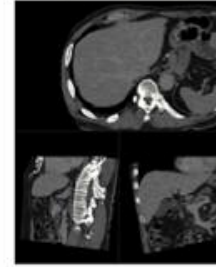

Application and repeated update of conversion parameters for non-rigid registration $\square . . \square$

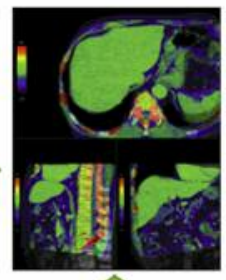

1

CT images scanned during expiration after optimal non-rigid registration

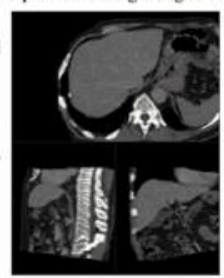

Generation of deformation vector field

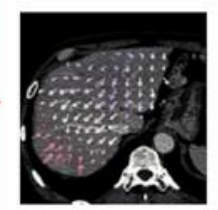


Table I. Correlation coefficients (Spearman's rho) between each computed tomographic (CT) parameter and the degree of liver fibrosis or grade of necro-inflammation.

\begin{tabular}{lccccc}
\hline & \multicolumn{2}{c}{ Fibrosis } & & \multicolumn{2}{c}{ Necro-inflammation } \\
\cline { 2 - 3 } \cline { 6 - 6 } CT parameter & Rho & $p$-Value & & Rho & $p$-Value \\
\hline Mean length & -0.101 & 0.50 & & -0.092 & 0.54 \\
SD & -0.317 & 0.030 & & -0.209 & 0.16 \\
CV & -0.376 & 0.009 & & -0.262 & 0.075 \\
Kurtosis & -0.254 & 0.085 & & -0.228 & 0.12 \\
Skewness & -0.306 & 0.037 & & -0.243 & 0.10 \\
95th Percentile value & -0.206 & 0.16 & & -0.138 & 0.36 \\
90th Percentile value & -0.136 & 0.36 & & -0.086 & 0.57 \\
80th Percentile value & -0.098 & 0.51 & & -0.079 & 0.60 \\
\hline
\end{tabular}

SD: Standard deviation; CV: coefficient of variance (=SD/mean length). The 95th, 90th and 80th percentile values represent the point at which 95,90 and $80 \%$ of the deformation vector length for all voxels are found to the left in the histogram.

expiration. The equilibrium phase during expiration was systematically acquired as a part of the hepatic CT protocol.

Image analysis. The 3D volume data of the portal venous phase scanned during inspiration and the equilibrium phase scanned during expiration were utilized for image analysis. The analysis was performed on an offline personal computer with original software developed and programmed for this analysis. Firstly, contour extraction of the liver was performed using CT images scanned during inspiration and expiration. One data analyst, who was unaware of the pathological results, evaluated the accuracy of contour extraction. Secondly, the rigid registration (only parallel shift and rotation) of the liver between CT images scanned during inspiration and expiration was performed. However, the livers extracted from the CT images scanned during inspiration and expiration still showed a morphological difference at this stage. Subsequently, non-rigid registration of the liver between the CT images scanned during inspiration and expiration was performed based on B-spline and diffeomorphic conversion models (14). The maximal coincidence in morphology of the livers extracted from the CT images scanned during inspiration and expiration was obtained in the last stage. The amount and direction of shift of each voxel during this non-rigid registration was defined as a deformation vector. A deformation vector field, comprising deformation vectors for all voxels, was generated. Details of image analysis are summarized in Figure 1.

Several feature values in relation to these deformation vectors originating from all voxels (potential CT parameters as indicators of stiffness) were obtained for analysis. These feature values (CT parameters) included mean length, standard deviation (SD), coefficient of variance ( $\mathrm{CV}=\mathrm{SD} /$ mean length), kurtosis, skewness, 95th percentile value, 90 th percentile value and 80 th percentile value of deformation vectors, and were automatically measured with the original software. The 95th, 90th and 80th percentile values represent the point at which 95,90 and $80 \%$ of the deformation vector length for all voxels are found to the left in the histogram.

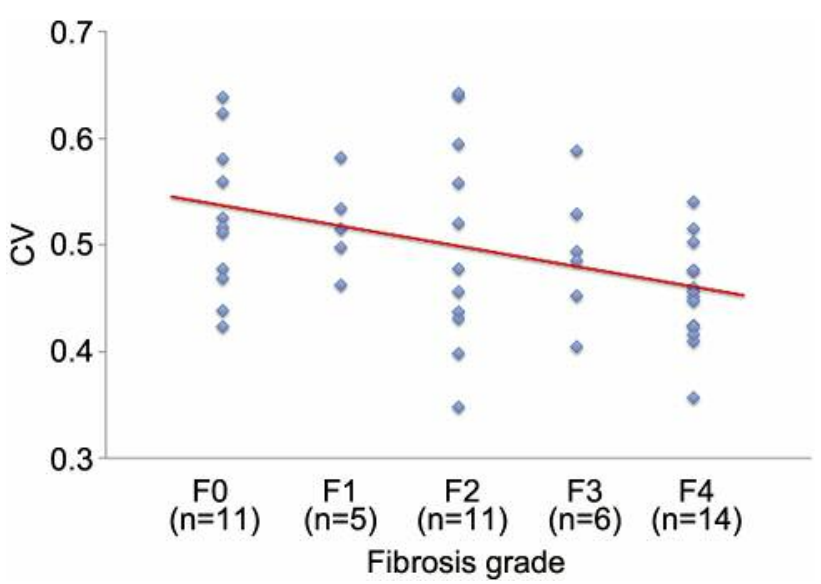

Figure 2. Correlation of coefficient of variance $(\mathrm{CV})$ with the degree of liver fibrosis. CV significantly decreased as liver fibrosis progressed (rho=-0.376) ( $p=0.009)$. The average $C V$ values for $F 0, F 1, F 2, F 3$ and F4 were $0.52 \pm 0.07,0.52 \pm 0.04,0.50 \pm 0.10,0.49 \pm 0.06$ and $0.45 \pm 0.05$, respectively.

Pathological analysis. One pathologist with 12 years of experience, who was unaware of the imaging data, reviewed the hematoxylineosin-stained glass slides for each patient and referred to the official pathological report to determine the histological findings of the liver parenchyma. The degree of liver fibrosis was classified into five groups according to the New Inuyama Classification (15): F0: no fibrosis, F1: fibrous portal expansion, F2: bridging fibrosis, F3: bridging fibrosis with architectural distortion, and F4: liver cirrhosis. Similarly, the grade of necro-inflammatory activity was scored as A0: none, A1: mild, A2: moderate, and A3: severe.

Statistical analysis. The correlations of the potential CT parameters as indicators of stiffness with the degree of liver fibrosis and the grade of necro-inflammatory activity were assessed using Spearman's rank correlation test, and a correlation coefficient (rho) was obtained.

For each CT parameter with the significant and highest correlation coefficient for the degree of liver fibrosis or the grade of necro-inflammatory activity, we also evaluated its ability to predict the histological findings of the liver parenchyma. Receiver operating characteristic (ROC) curve analysis was conducted for prediction of F1 or greater, F2 or greater, F3 or greater and F4 fibrosis; or for the prediction of $\mathrm{A} 1$ or greater, $\mathrm{A} 2$ or greater and $\mathrm{A} 3$ necro-inflammation. The feasible cut-off point of the CT parameter was determined using the point on the curve closest to the 0,1 point (10). The sensitivity was defined as the percentage of positive cases for which each CT parameter was at or smaller than the given threshold level, while the specificity was defined as the percentage of negative cases for which each CT parameter was greater than the given threshold level. The positive predictive value, negative predictive value, and accuracy were also calculated.

Discriminant analysis was also performed using all CT parameters that were significantly correlated with the degree of liver fibrosis or the grade of necro-inflammatory activity. This discriminant analysis was conducted for prediction of F1 or greater, F2 or greater, F3 or greater and F4 fibrosis; and for prediction of A1 or greater, A2 or greater and A3 necro-inflammation. Correlation ratios and Wilks' 
Table II. Diagnostic performance of coefficient of variance (CV) for prediction of the degree of liver fibrosis using receiver operating curve analysis.

\begin{tabular}{lcccc}
\hline & \multicolumn{4}{c}{ Prediction of } \\
\cline { 2 - 5 } Parameter & $\geq \mathrm{F} 1$ & $\geq \mathrm{F} 2$ & $\geq \mathrm{F} 3$ & \multirow{2}{*}{$\mathrm{F} 4$} \\
\hline AUC & $0.654(0.475-0.833)$ & $0.690(0.536-0.843)$ & $0.689(0.538-0.840)$ & $0.727(0.581-0.873)$ \\
CV cutoff & 0.502 or less & 0.493 or less & 0.493 or less & 0.476 or less \\
Sensitivity (\%) & $66.7(50.3-79.8)$ & $67.7(50.1-81.4)$ & $75.0(53.1-88.8)$ & $78.6(52.4-92.4)$ \\
Specificity (\%) & $63.6(35.4-84.8)$ & $68.8(44.4-85.8)$ & $63.0(40.7-75.5)$ & $66.7(49.6-80.3)$ \\
Accuracy (\%) & 66.0 & 68.1 & 68.1 & 70.2 \\
PPV (\%) & 85.7 & 80.8 & 60.0 & 50.0 \\
NPV (\%) & 36.8 & 52.4 & 77.3 & 88.0 \\
\hline
\end{tabular}

AUC: Area under the receiver operating characteristics curve; CV: standard deviation/mean length; PPV: positive predictive value; NPV: negative predictive value. Numbers in parentheses represent the $95 \%$ confidence interval.

lambda were measured, and sensitivity, specificity, accuracy, positive predictive value, and negative predictive value were calculated.

For all tests, $p$-values of less than 0.05 were taken to indicate statistically significant differences.

\section{Results}

The results of pathological analysis for the liver parenchyma were as follows: $F 0: n=11, F 1: n=5, F 2: n=11, F 3: n=6, F 4$ : $\mathrm{n}=14$, and A0: $\mathrm{n}=4, \mathrm{~A} 1: \mathrm{n}=25, \mathrm{~A} 2: \mathrm{n}=18, \mathrm{~A} 3: \mathrm{n}=0$. Table I shows the correlation coefficients between each CT parameter and the degree of liver fibrosis or the grade of necroinflammatory activity. The $\mathrm{SD}, \mathrm{CV}$ and skewness were significantly negatively correlated with the degree of liver fibrosis. Of them, CV was best correlated with the degree of liver fibrosis and significantly decreased as liver fibrosis progressed (rho $=-0.376$ ) (Figure 2). The average $\mathrm{CV}$ values of $\mathrm{F} 0, \mathrm{~F} 1, \mathrm{~F} 2, \mathrm{~F} 3$ and F4 cases were $0.52 \pm 0.07,0.52 \pm 0.04$, $0.50 \pm 0.10,0.49 \pm 0.06$ and $0.45 \pm 0.05$, respectively. On the other hand, no significant correlation was obtained between any CT parameter and the grade of necro-inflammatory activity.

The results of ROC analyses for prediction of the degree of liver fibrosis for $\mathrm{CV}$ are summarized in Table II. CV showed an accuracy of 66.0 to $70.2 \%$, and the areas under curves were 0.654 to 0.727 for prediction of $\mathrm{F} 1$ or greater, F2 or greater, F3 or greater and F4 fibrosis.

The results of discriminant analyses using SD, CV and skewness for prediction of the degree of liver fibrosis are summarized in Table III. A combination of SD, CV and skewness showed an accuracy of 66.0 to $72.3 \%$ for prediction of F1 or greater, F2 or greater, F3 or greater and F4 fibrosis.

Representative cases are shown in Figures 3 and 4.

\section{Discussion}

In this study, the SD, CV and skewness of the deformation vectors were correlated with the degree of liver fibrosis and significantly decreased as liver fibrosis progressed. When an
Table III. Diagnostic performance of standard deviation (SD), coefficient of variance $(\mathrm{CV})$ and skewness for prediction of the degree of liver fibrosis using a discriminant analysis.

\begin{tabular}{lcccc}
\hline & \multicolumn{4}{c}{ Prediction of } \\
\cline { 2 - 5 } Parameter & $\geq \mathrm{F} 1$ & $\geq \mathrm{F} 2$ & $\geq \mathrm{F} 3$ & $\mathrm{~F} 4$ \\
\hline Correlation ratio & 0.214 & 0.190 & 0.160 & 0.156 \\
Wilks' lambda & 0.786 & 0.810 & 0.840 & 0.846 \\
$p$-Value & 0.015 & 0.027 & 0.056 & 0.063 \\
Sensitivity (\%) & 75.0 & 77.4 & 80.0 & 85.7 \\
Specificity (\%) & 63.6 & 56.3 & 55.6 & 60.6 \\
Accuracy (\%) & 72.3 & 70.2 & 66.0 & 68.1 \\
PPV (\%) & 87.1 & 77.4 & 57.1 & 48.0 \\
NPV (\%) & 43.8 & 56.3 & 78.9 & 90.9 \\
\hline
\end{tabular}

CV: SD/mean length; PPV: positive predictive value; NPV: negative predictive value.

external force is given to any structure, the corresponding area is supposed to be generally deformed. Differences in the lengths of deformation vectors between deformed and undeformed areas would increase in a soft liver with less fibrosis compared to a stiffer liver with more fibrosis. Indeed, the $\mathrm{CV}$ of the deformation vector was found to be best correlated with the degree of liver fibrosis and showed fairly high diagnostic ability for liver fibrosis (accuracy: 66-70.2\%). Because CV is a standardized parameter that does not depend on a patient's liver size, this result seems reasonable. By contrast, the mean length, and 95th, 90th and 80th percentile values of the deformation vector were not significantly correlated with the degree of liver fibrosis. These parameters are absolute values affected by a person's liver size. Thus, the CV of the deformation vector may be an appropriate and convenient parameter to indicate stiffness and evaluate liver fibrosis.

Watanabe et al. developed MR elastography with cinetagging and bending energy analysis. That study also utilized deformity of the liver during respiration. They concluded that 
A

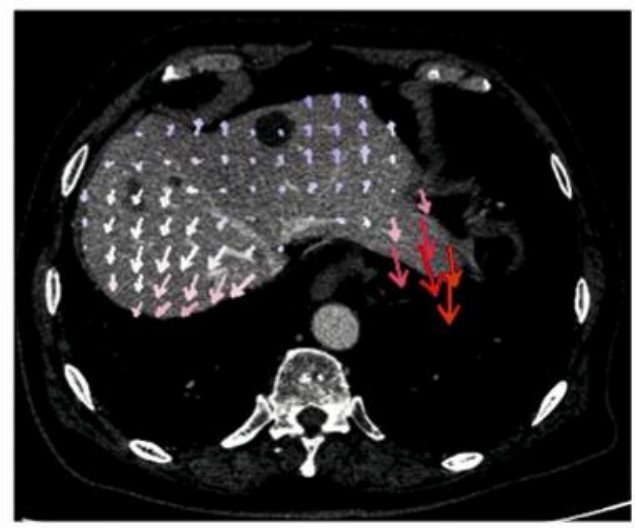

B

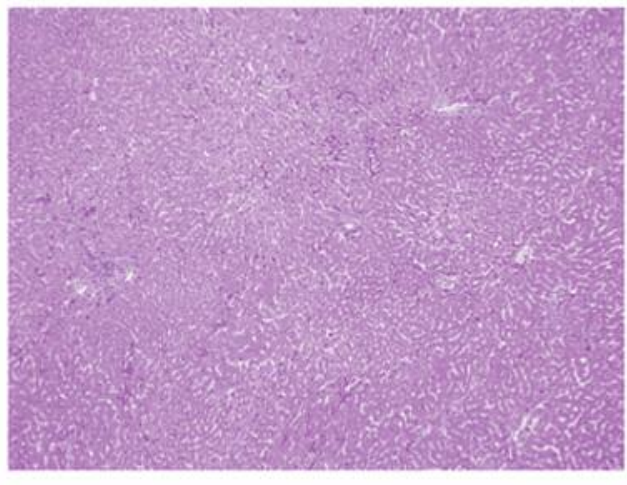

Figure 3. Non-rigid registration of computed tomographic (CT) imaging of a 70-year-old man with a liver metastasis. The Child-Pugh classification was grade A. A: Deformation vector field. Deformation vectors were simply described at intervals of $1.5 \mathrm{~cm}$. Color represents the direction of the deformation vector. Red, Shift to the cranial direction; Blue, shift to the caudal direction. Standard deviation=3.334; coefficient of variance=0.638; skewness=3.582. B: Hematoxylin-eosin staining (magnification, $\times 20$ ). The degree of liver fibrosis was F0, while the grade of necro-inflammatory activity was A1.

A

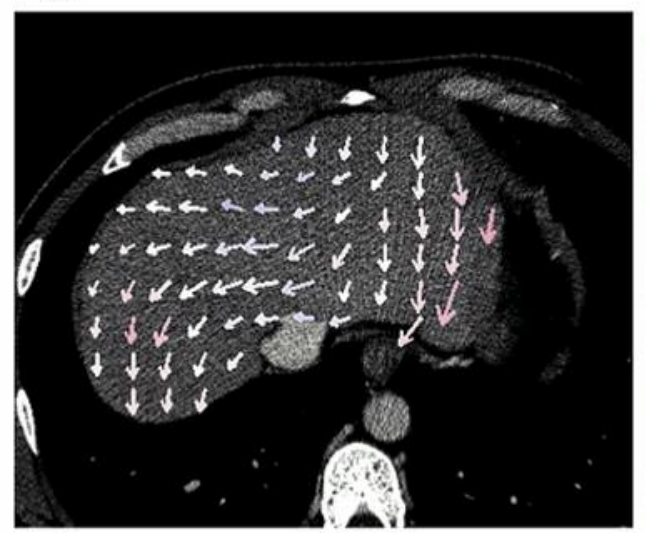

B

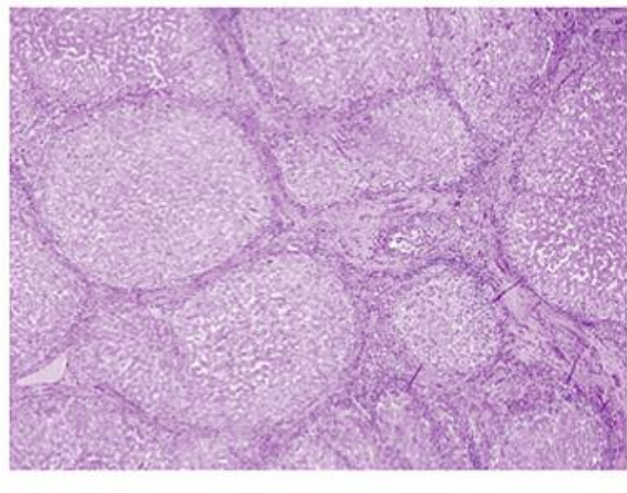

Figure 4. Non-rigid registration of computed tomographic (CT) imaging of a 39-year-old man with alcoholic liver disease. The Child-Pugh classification was grade B. A: Deformation vector field. Deformation vectors were simply described at intervals of $1.5 \mathrm{~cm}$. Color represents the direction of the deformation vectors. Red, shift to the cranial direction; Blue, shift to the caudal direction. Standard deviation=1.972; coefficient of variance=0.357; skewness=0.488. B: Hematoxylin-eosin staining (magnification, $\times 20$ ). The degree of liver fibrosis was F4, while the grade of necro-inflammatory activity was A2.

assessment with a sagittal grid and bending-energy analysis showed potential for discriminating patients with moderate or advanced liver fibrosis from those with healthy liver or slight fibrosis (13). This result is based on the fact that the posterobasal segments of the lung most actively deflate and shrink during forced exhalation and that such large motions more directly affect the dorsal segments of the liver. However, fibrosis is often unevenly distributed in the liver. Because the deformation vectors from the entire liver are automatically assessed in our method, it has the comparative advantage of considering information from the whole liver.
According to a previous meta-analysis, the pooled sensitivity and specificity using MR elastography for the diagnosis of F2 or greater, F3 or greater and F4 were $92.8 \%$ and $93.7 \%, 89.6 \%$ and $93.2 \%, 89.5 \%$ and $92.0 \%$, respectively. In contrast, the corresponding pooled sensitivity and specificity using Fibroscan (US elastography) were $71.6 \%$ and $81.6 \%, 79.0 \%$ and $84.6 \%, 80.0 \%$ and $86.6 \%$, respectively (16). In both methods, liver stiffness is estimated based on the measurement of shear-wave velocity. Unfortunately, the diagnostic performance of our method with CT may be inferior to those of MR and US 
elastography in spite of our use of a discriminant analysis using SD, CV and skewness that did not show significant improvement in diagnostic accuracy compared with use of CV only. The reason why a discriminant analysis did not improve the diagnostic performance is possibly due to the fact that SD, CV and skewness are all factors related to heterogeneity in length of deformation vectors. Although in practice its use alone may be limited at present, the deformation vector is a potential CT parameter for evaluating liver fibrosis. Naganawa et al. recently reported that CT texture analysis effectively predicted non-alcoholic steatohepatitis (17). A combination of deformation vectors and texture analysis for liver parenchyma may enhance the diagnostic performance of our method for the prediction of liver fibrosis.

Our study had several limitations. The first was the difference in the degree of inspiration as an external force among patients. It was extremely difficult to obtain similar external force for each patient's liver only with breathing instructions. We did exclude patients who had especially poor respiratory control, that is, less than $2 \mathrm{~cm}$ difference in the height of the top of the diaphragm between inspiration and expiration. Secondly, the proportion of patients suitable for this evaluation method may be limited. It would be difficult to assess patients with history of major liver or lung resection, such as lobectomy, because the external force from inspiration may not be sufficiently transmitted to the liver. We excluded such patients from this study. Thirdly, accurate contour extraction of the liver was incomplete in some cases, especially for the equilibrium phase. This limitation would be improved by using late arterial-phase images instead of equilibrium-phase images. Fourthly, because the tumors were recognized as a part of the liver parenchyma, they might affect the values of the potential CT parameters. It was difficult to exclude tumorous lesions specifically for the image analysis.

In conclusion, the deformation vector is a potential CT parameter for evaluating liver fibrosis.

\section{Acknowledgements}

The Authors thank Professor Yoshinao Oda, Department of Anatomic Pathology, Kyushu University for providing pathological information for this study.

\section{Funding}

No grant or fund supported this work.

\section{Conflict of Interest}

Sadato Akahori and Yuanzhong Li are employees of FUJIFILM Corporation. The other Authors declare that they have no conflict of interest in regard to this study.

\section{Authors' Contributions}

Study concepts: Nishie A. Study design: Nishie A and Akahori S. Data acquisition: Nishie A, Kohashi K, Yoshizumi T and Li Y. Quality control of data and algorithms: Asayama Y, Ishigami K, Ushijima Y and Ishimatsu K. Data analysis and interpretation: Takayama Y, Fujita N, Morita K and Takao S. Statistical analysis: Nishie A, Ushijima Y, Kakahara D and Nakayama T. Manuscript preparation: Nishie A and Akahori S. Manuscript editing: Honda H. Manuscript review: All Authors.

\section{References}

1 Kim AI and Saab S: Treatment of hepatitis C. Am J Med 118: 808-815, 2005. PMID: 16084169. DOI: 10.1016/j.amjmed. 2005.01.073

2 Kumada $\mathrm{H}$, Okanoue $\mathrm{T}$, Onji $\mathrm{M}$, Moriwaki $\mathrm{H}$, Izumi $\mathrm{N}$, Tanaka E, Chayama K, Sakisaka S, Takehara T, Oketani M, Suzuki F, Toyota J, Nomura H, Yoshioka K, Seike M, Yotsuyanagi H, Ueno Y; Study Group for the Standardization of Treatment of Viral Hepatitis Including Cirrhosis, Ministry of Health, Labour and Welfare of Japan: Guidelines for the treatment of chronic hepatitis and cirrhosis due to hepatitis B virus infection for the fiscal year 2008 in Japan. Hepatol Res 40: 1-7, 2010. PMID: 20156295. DOI: $10.1111 / \mathrm{j} .1872-$ 034X.2009.00633.x

3 Kumada H, Okanoue T, Onji M, Moriwaki H, Izumi N, Tanaka E, Chayama K, Sakisaka S, Takehara T, Oketani M, Suzuki F, Toyota J, Nomura H, Yoshioka K, Seike M, Yotsuyanagi H, Ueno Y; Study Group for the Standardization of Treatment of Viral Hepatitis Including Cirrhosis, Ministry of Health, Labour and Welfare of Japan: Guidelines for the treatment of chronic hepatitis and cirrhosis due to hepatitis $\mathrm{C}$ virus infection for the fiscal year 2008 in Japan. Hepatol Res 40: 8-13, 2010. PMID: 20156296. DOI: 10.1111/j.1872-034X.2009.00634.X

4 Perz JF, Armstrong GL, Farrington LA, Hutin YJ and Bell BP: The contributions of hepatitis $\mathrm{B}$ virus and hepatitis $\mathrm{C}$ virus infections to cirrhosis and primary liver cancer worldwide. J Hepatol 45: 529-538, 2006. PMID: 16879891. DOI: 10.1016/ j.jhep.2006.05.013

5 Petitclerc L, Sebastiani G, Gilbert G, Cloutier G and Tang A: Liver fibrosis: Review of current imaging and MRI quantification techniques. J Magn Reson Imaging 45: 12761295, 2017. PMID: 27981751. DOI: 10.1002/jmri.25550

6 Goshima S, Kanematsu M, Kobayashi T, Furukawa T, Zhang X, Fujita H, Watanabe H, Kondo H, Moriyama N and Bae KT: Staging hepatic fibrosis: Computer-aided analysis of hepatic contours on gadolinium ethoxybenzyl diethylenetriaminepentaacetic acid-enhanced hepatocyte-phase magnetic resonance imaging. Hepatology 55: 328-329, 2012. PMID: 21932401. DOI: $10.1002 /$ hep. 24677

7 Vicas C, Lupsor M, Badea R and Nedevschi S: Usefulness of textural analysis as a tool for noninvasive liver fibrosis staging. J Med Ultrason 38: 105-117, 2011. PMID: 27278498. DOI: 10.1007/s10396-011-0307-x

8 Ou HY, Bonekamp S, Bonekamp D, Corona-Villalobos CP, Torbenson MS, Geiger B and Kamel IR: MRI arterial enhancement fraction in hepatic fibrosis and cirrhosis. Am J Roentgenol 201: W596-602, 2013. PMID: 24059398. DOI: 10.2214/AJR.12.10048 
9 Wang QB, Zhu H, Liu HL and Zhang B: Performance of magnetic resonance elastography and diffusion-weighted imaging for the staging of hepatic fibrosis: A meta-analysis. Hepatology 56: 239247, 2012. PMID: 22278368. DOI: 10.1002/hep.25610

10 Nishie A, Asayama Y, Ishigami K, Tajima T, Kakihara D, Nakayama T, Takayama Y, Okamoto D, Taketomi A, Shirabe K, Fujita N, Obara M, Yoshimitsu K and Honda H: MR prediction of liver fibrosis using a liver-specific contrast agent: Superparamagnetic iron oxide versus Gd-EOB-DTPA. J Magn Reson Imaging 36: 664-671, 2012. PMID: 22532503. DOI: 10.1002/jmri.23691

11 Kennedy P, Wagner M, Castéra L, Hong CW, Johnson CL, Sirlin $\mathrm{CB}$ and Taouli B: Quantitative elastography methods in liver disease: Current evidence and future directions. Radiology 286: 738-763, 2018. PMID: 29461949. DOI: 10.1148/radiol.201817 0601

12 Shimizu Y, Takamatsu S, Yamamoto K, Maeda Y, Sasaki M, Tamamura H, Bou S, Kumano T and Gabata T: Segmental analysis of respiratory liver motion in patients with and without a history of abdominal surgery. Jpn J Radiol, 2018. PMID: 29922899. DOI: 10.1007/s11604-018-0750-3

13 Watanabe H, Kanematsu M, Kitagawa T, Suzuki Y, Kondo H, Goshima S, Kajita K, Bae KT, Hirose Y, Miotani S, Zhou X and Fujita H: MR elastography of the liver at $3 \mathrm{~T}$ with cine-tagging and bending energy analysis: preliminary results. Eur Radiol 20: 23812389, 2010. PMID: 20440504. DOI: 10.1007/s00330-010-1800-0
14 Mattes D, Haynor DR, Vesselle H, Lewellyn TK and Eubank W: Nonrigid multimodality image registration. Proc SPIE 4322, Medical Imaging 2001. DOI: 10.1117/12.431046

15 Tomimaru Y, Sasaki Y, Yamada T, Eguchi H, Ohigashi H, Ishikawa $\mathrm{O}$ and Imaoka $\mathrm{S}$ : Fibrosis in non-cancerous tissue is the unique prognostic factor for primary hepatocellular carcinoma without hepatitis B or C viral infection. World J Surg 30: 1729-1735, 2006. PMID: 16850156. DOI: 10.1007/s00268005-0123-9

16 Xiao H, Shi M, Xie Y and Chi X: Comparison of diagnostic accuracy of magnetic resonance elastography and Fibroscan for detecting liver fibrosis in chronic hepatitis B patients: A systematic review and meta-analysis. PLoS One 12: e0186660, 2017. PMID: 29107943. DOI: 10.1371/journal.pone.0186660

17 Naganawa S, Enooku K, Tateishi R, Akai H, Yasaka K, Shibahara J, Ushiku T, Abe O, Ohtomo K and Kiryu S: Imaging prediction of nonalcoholic steatohepatitis using computed tomography texture analysis. Eur Radiol 28: 3050-3058, 2018. PMID: 29404772. DOI: 10.1007/s00330-017-5270-5

Received January 28, 2019

Revised February 16, 2019

Accepted February 20, 2019 